\title{
Ökonomische Folgen von Pandemien
}

\author{
Clemens Fuest ${ }^{(\bowtie)}$ \\ Ifo Institut, Leibniz-Institut für Wirtschaftsforschung an der Universität \\ München, München, Deutschland \\ fuestaifo.de
}

Schlüsselwörter: Pandemie $\cdot$ Rezession $\cdot$ Gesundheit $\cdot$ Zielkonflikt

\section{Einleitung}

$\mathrm{Zu}$ den ökonomischen Folgen von Pandemien gehören:

- Mehraufwand für das Gesundheitswesen, beispielsweise für Medikamente, Tests und Krankenhauskapazitäten

- Unterbrechungen von Wirtschaftstätigkeit und Lieferketten, unter anderem wegen Lockdowns oder eingeschränkter Produktion aufgrund von Arbeitnehmerschutz und Krankheitsausfällen. Während Stilllegungen fällt schnell eine sehr große Wertschöpfung aus: Wenn man für einen Monat die Hälfte der gesamtwirtschaftlichen Wertschöpfung in Deutschland lahmlegt, bricht die jährliche Wirtschaftsleistung um vier Prozentpunkte ein. Was das heißt, wird deutlich, wenn man es mit den üblichen jährlichen deutschen Wachstumsraten von eins bis drei Prozent vergleicht: Die Stilllegung in diesem Beispiel führt innerhalb eines Monats dazu, dass die Wirtschaft im Jahr nicht um zwei Prozent wächst, sondern um zwei Prozent einbricht.

- Geringer sozialer Konsum, weil Aktivitäten eingeschränkt sind, bei denen sich Menschen treffen. Damit fallen Veranstaltungen aus, Hotels und Restaurants stehen leer, die Reisebranche bricht ein.

- Wenn der Staat im Laufe einer Pandemie Schulden macht, kann sich das mittelfristig negativ auf den Kapitalmarkt und die Geldwertstabilität auswirken.

- Arbeitsplätze, Einkommen, wirtschaftliche Existenz und Finanzstabilität sind gefährdet. Ökonomisch sehr wichtig ist die Frage, ob es zu einer Abwärtsspirale kommt: Mündet die Pandemie in eine Finanzkrise, etwa weil Banken Forderungen verlieren und Kredite nicht bedient werden?

Ein Blick auf historische Pandemien belegt die wirtschaftlichen Folgen. Nach der Pestepidemie im 14. Jahrhundert, an der sehr viele Menschen aus der ganzen Bevölkerung starben, wurden die Arbeitskräfte knapp, das Wachstum ging stark zurück, die Löhne stiegen und die Kapitalentlohnung fiel [1]. Als nach dem Ersten Weltkrieg die Spanische Grippe kursierte, brach die Wirtschaftsleitung in vielen Ländern geschätzt um sechs bis acht Prozent ein [2]. Allerdings sind diese Schätzungen mit einer recht großen Unsicherheit behaftet, denn der Erste Weltkrieg und seine Folgen beeinflussten ebenfalls die Wirtschaftsleistung. 


\section{Einordnung der COVID-19-Rezession}

Die Rezession aufgrund der COVID-19-Pandemie lässt sich einordnen, indem man sie mit den bedeutendsten anderen Wirtschaftskrisen in den letzten hundert Jahren vergleicht: die Finanzkrise vor rund zehn Jahren und die weltweite Depression der 1930-er Jahre. Abb. 1 zeigt die Entwicklung des ungewichtet kumulierten Bruttoinlandsprodukts (BIP) von vier Ländern - USA, Großbritannien, Frankreich und Deutschland - während dieser Krisen. In der Finanzkrise brach dieses kumulierte BIP innerhalb eines Jahres um rund vier Prozent ein. Doch schon im Jahr darauf erholte sich die Wirtschaft wieder. Die Rezession in den 1930-er Jahren hatte eine andere Größenordnung: Da brach das BIP über mehrere Jahre hinweg massiv bis zu acht Prozent jährlich ein.

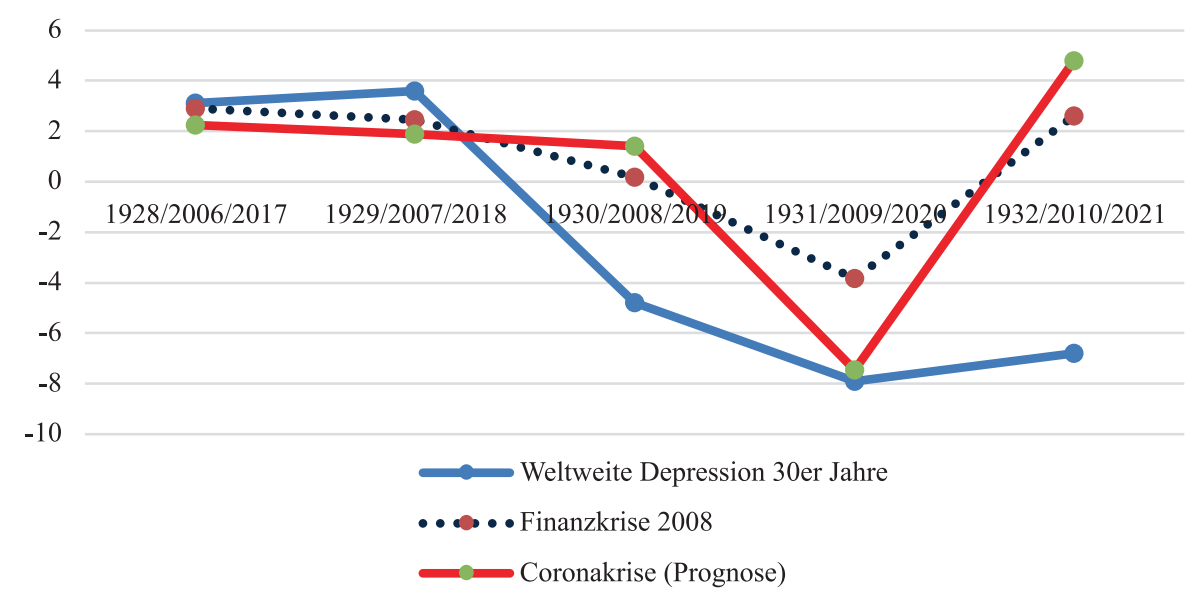

Quelle: Fuest (2020), Maddison Project, Ritschl und Spoerer (1997); IWF WEO, April 2020; eigene Berechnungen.

Abb. 1. Vergleich des Wirtschaftswachstums in Wirtschaftskrisen. (Veränderung des BIP gegenüber Vorjahr in Prozent; ungewichteter Durchschnitt von Deutschland, Frankreich, Vereinigtes Königreich, USA)

Die derzeitige COVID-19-Krise ist noch in einem frühen Stadium. Insofern kann der Vergleich mit den beiden genannten historischen Krisen nur auf einer Prognose beruhen. In Konjunkturprognosen erwartet das ifo Institut für 2020 für die vier Länder eine Rezession in einer Dimension wie in den 1930-er Jahren [3]. Doch es geht davon aus, dass sich die Wirtschaft rasch und schon 2021 deutlich erholen wird. Das heißt, die COVID-19-Pandemie wirkt sich wirtschaftlich stärker aus als die Finanzkrise, ist aber nicht so dramatisch wie die große Depression vor dem Zweiten Weltkrieg. Allerdings lässt sich derzeit noch nicht absehen, wie sich die sogenannte zweite Welle der Pandemie auswirken wird, die offensichtlich gerade anrollt.

Aus der Sicht des Arbeitsmarktes ist bereits jetzt zu erkennen, dass die COVID19-Krise größer ist als die Finanzkrise. Abb. 2 zeigt die Zahl der deutschen 
Arbeitnehmer, die jeweils von Kurzarbeit betroffen waren. Die Kurzarbeit war während und nach der Finanzkrise in aller Munde: Man sprach darüber, dass die Kurzarbeit viel zur Bewältigung der Krise beigetragen hat. Verglichen mit den Anzeigen für Kurzarbeit während der COVID-19-Pandemie, waren die damaligen Zahlen allerdings sehr niedrig.

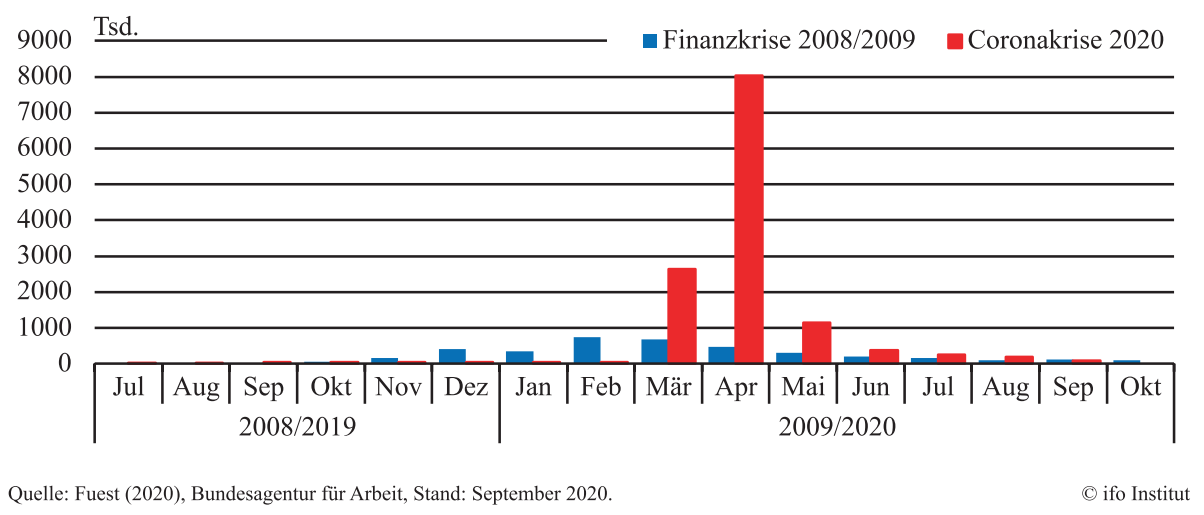

Abb. 2. Anzeigen zur Kurzarbeit in Deutschland (betroffene Arbeitnehmer)

Es gibt einen beachtenswerten Aspekt der COVID-19-Rezession: Das verfügbare Einkommen der Privathaushalte ist aufgrund der Hilfsmaßnahmen des Staates kaum gesunken. In herkömmlichen Wirtschaftskrisen bricht dagegen das verfügbare Einkommen meist ein, was dann zu einem stark sinkenden privaten Konsum führt. In der aktuellen Krise ist der Konsum dagegen aus anderen Gründen eingebrochen: Man kann nicht mehr in Urlaub fahren, ins Kino gehen oder im Restaurant essen. Das bedeutet: Anders als in anderen Wirtschaftskrisen haben die Menschen eigentlich Geld - sie geben es nur nicht aus. Für die Wirtschaftspolitik heißt das: Um die Krise zu bewältigen, bringt es wenig, die verfügbaren Einkommen in die Höhe zu bringen oder den Menschen Mittel zu geben, damit sie konsumieren können. Die herkömmlichen Maßnahmen, mit denen die Nachfrage erhöht wird, laufen ins Leere, zumindest, solange die Pandemie nicht beendet ist.

Ein zweiter wichtiger Aspekt ist, dass die COVID-19-Krise verschiedene Branchen sehr unterschiedlich trifft [4] - anders als in manchen herkömmlichen Wirtschaftskrisen. Besonders leiden Reisebüros und Reiseveranstalter und das Gastgewerbe. Aber es gab auch Industriesektoren wie den Maschinenbau oder die Chemieproduktion, die am Anfang der Pandemie stark betroffen waren. Ursache waren die Grenzschließungen und die Zerschlagung der Wertschöpfungsketten. Für die Politik bedeutet das: Um zu helfen, muss man sehr gezielt vorgehen, anstatt auf flächendeckende Instrumente zu setzen. 


\section{Verlauf der COVID-19-Rezession}

Abb. 3 zeigt die Entwicklung des ifo Geschäftsklimaindex für Deutschland von 2007 bis Oktober 2020. Dieser Index ist ein Konjunkturindikator, den das ifo Institut monatlich ermittelt und veröffentlicht. Er beruht auf den Daten von 9000 deutschen Unternehmen. $\mathrm{Zu}$ erkennen ist, dass sich die Wirtschaft gegenüber dem Anfang der COVID-19-Pandemie erholt hat. Allerdings ist der starke Anstieg der Geschäftserwartung vorsichtig zu bewerten: Wenn man keinen Umsatz macht, dann kann es eigentlich nur noch besser werden. Deshalb steigen die Werte für die Erwartungen gleichsam automatisch, nachdem die Werte für die Wirtschaftslage stark gefallen sind. Der Lageindikator ist dagegen aussagekräftiger: Er zeigt, dass die deutsche Wirtschaft nach dem Pandemie-bedingten Absturz bis heute rund die Hälfte des Verlustes wieder aufgeholt hat. Trotzdem ist die Wirtschaft weit davon entfernt, wieder auf dem Niveau vor der Krise zu sein.
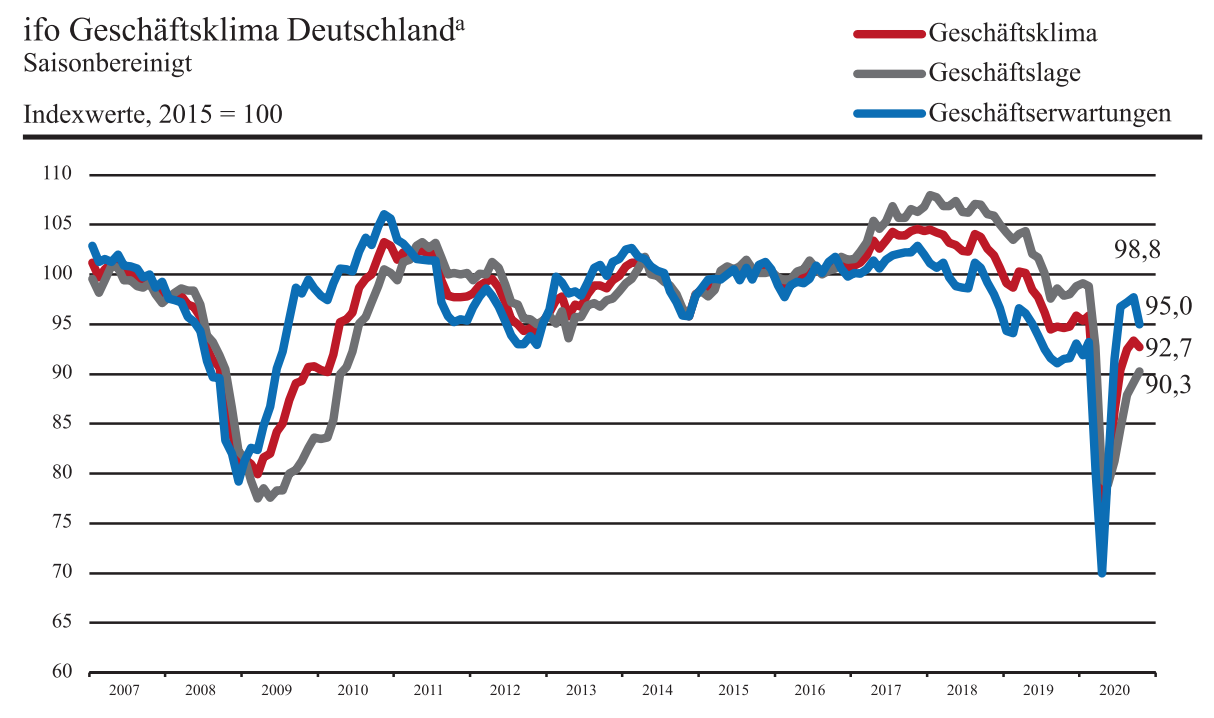

a Verarb eitendes Gewerbe, Dienstleistungssektor, Handel und Bauhauptgewerbe. Quelle: ifo Konjunkturumfragen, Oktober 2020.

Abb. 3. Ifo Geschäftsklima für Deutschland, 2007 bis 2020

Beim Export zeigt sich ein ähnliches Bild. Der Exportumsatz liegt deutlich unter dem des Vorjahres. Im April 2020 betrug er rund 76 Mrd. EUR - rund 35 Mrd. weniger als im April 2019 [5]. Im August war die Differenz zum Vorjahr mit rund 10 Mrd. EUR weniger groß, sodass man sagen kann, auch die Exporte haben sich erholt. Inzwischen exportiert Deutschland nach China bereits wieder mindestens so viel wie vor der Pandemie, doch die Exporte in europäische Länder und die USA sind noch beeinträchtigt. 
Andere Indikatoren zur Wirtschaftsaktivität weisen in die gleiche Richtung. So kann man beispielsweise die Mobilitätdaten untersuchen, etwa die Aufenthaltsdauer auf Bahnhöfen und Haltestellen. Da sind wir inzwischen auf 80 bis $90 \%$ des Niveaus vor der Krise.

Derzeit herrscht in Deutschland so etwas wie eine „90-Prozent-Ökonomie“. Denn ein Teil unserer Wirtschaft - sozialer Konsum, Reisen, Restaurants -, die vor der Krise rund sechs bis sieben Prozent des Bruttoinlandsproduktes ausmacht, ist nach wie vor stark beeinträchtigt und wird es sicherlich noch länger bleiben. Daher wird die deutsche Wirtschaft nicht so schnell wieder das Vorkrisenniveau erreichen.

Das ifo Institut hat mittels einer Simulation berechnet, wie viele Insolvenzen infolge der COVID-19-Pandemie zu erwarten sind (Abb. 4). Basis für die Simulation waren die Insolvenzen, die auf vergangene Wirtschaftskrisen folgten. Aus der Prognose geht hervor, dass wir vor einem Anstieg der Insolvenzen stehen. ${ }^{1} \mathrm{Ob}$ es tatsächlich so kommt, ist offen, eben weil die COVID-19-Krise ganz anders ist wie vorangegangene Wirtschaftskrisen.

Unternehmensinsolvenzen und Konjunktur

Konjunkturelle Komponente der Unternehmensinsolvenzen - - ifo Kapazitätsauslastung Gesamtwirtschaft

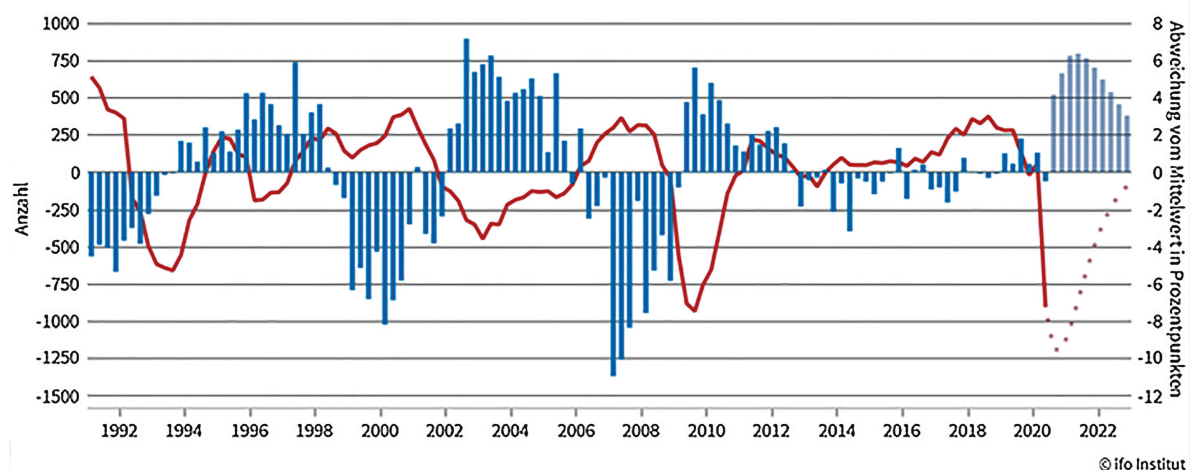

Abb. 4. Simulation der erwarteten Insolvenzen auf Basis von Zusammenhängen bei früheren Wirtschaftskrisen

\section{Konflikt Zwischen Gesundheit Und Wirtschaft?}

Es ist eine viel diskutierte Frage, ob Lockdowns, die zum Schutz der öffentlichen Gesundheit angeordnet werden, die wirtschaftliche Rezession verschlimmern. Oder andersherum ausgedrückt: Kann der Staat die Wirtschaft durch Öffnungspolitik trotz Pandemie beleben?

1 Liquiditätshilfen des Staates zur Abfederung der Coronakrise wurden dabei noch nicht berücksichtigt. Jüngere Simulationen des ifo Instituts aus ihrer Konjunkturprognose im Winter 2020 zeigen, dass die politischen Gegenmaßnahmen die Insolvenzwelle voraussichtlich deutlich abschwächen werden [6]. 


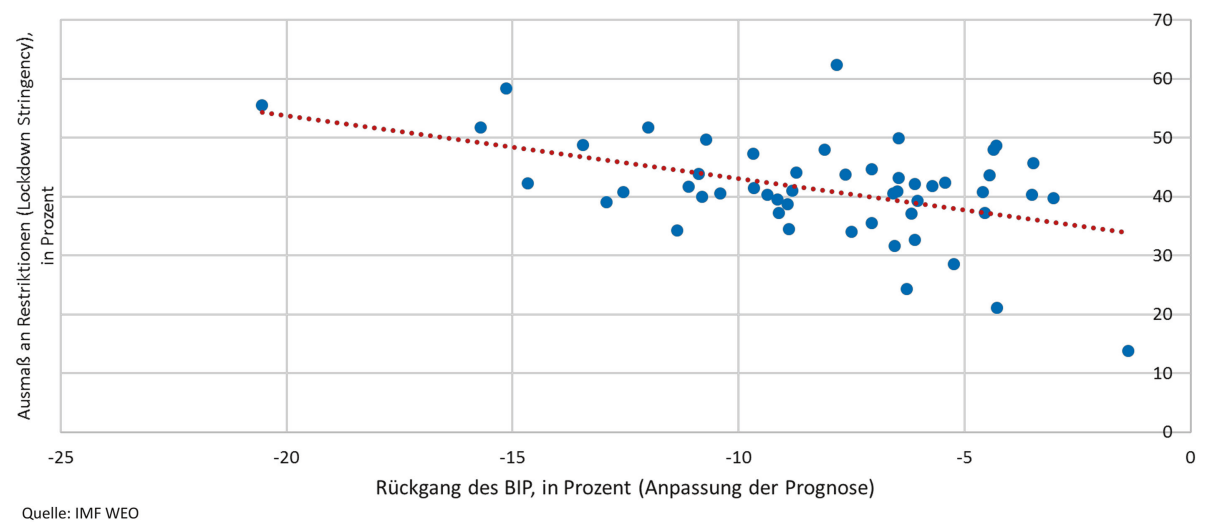

Abb. 5. Korrelation zwischen der Strenge des Lockdowns und dem Rückgang des Bruttoinlandsprodukts in verschiedenen Ländern

Abb. 5 zeigt, dass es eine Korrelation zwischen der Intensität von Lockdowns und der wirtschaftlichen Entwicklung gibt: In Ländern, die das öffentliche Leben besonders stark einschränken, ist der Einbruch des Bruttoinlandsprodukts größer. Daraus würden manche Menschen folgern, dass die Lockdowns tatsächlich die Ursache für den wirtschaftlichen Abschwung in der COVID-19-Pandemie sind, dass es also eine Kausalität gibt. Doch sie machen einen bekannten Denkfehler. Denn Korrelationen sagen nichts über Kausalitäten aus. Die Korrelation kann in diesem Fall beispielsweise auch dadurch zustande kommen, dass man bei hohen Infektionszahlen auch härtere Schutzmaßnahmen ergreift, dass also die beobachtete Korrelation auf eine Kausalität von Infektionszahlen und wirtschaftlicher Rezession zurückzuführen ist.

Eine Studie hat die Lockdown-Politik verschiedener Städte in den USA während der Spanischen Grippe im Jahr 1918 untersucht [7]. Die Ergebnisse, kurz zusammengefasst:

- In Städten, in denen besonders viele Menschen an Grippe und Lungenentzündung starben, gingen die wirtschaftlichen Aktivitäten besonders stark und lange zurück.

- Städte mit schnelleren und konsequenten Restriktionen verzeichneten weniger Tote und weniger negative Effekte auf die mittelfristige Wirtschaftsentwicklung als Städte, die weniger konsequent Maßnahmen ergriffen.

Aktuell wird viel über den schwedischen Weg diskutiert, mit der COVID-19Pandemie umzugehen. Dort gab es zwar auch Infektionsschutzmaßnahmen, jedoch keine landesweiten Ausgangssperren und Kontaktverbote. Die Behörden dort entschieden sich vorrangig, auf Empfehlungen und Appelle an die Vernunft der Menschen zu setzen. Der Frage, inwieweit die vergleichsweise hohe Zahl an Infizierten und Toten in Schweden eine Folge dieser Politik ist, soll hier nicht nachgegangen werden. Während etwa nach Angaben der Johns-Hopkins-Universität [8] - Stand 21.10.2020 - in Schweden pro 100000 Einwohner 58 COVID-19-Tote gemeldet wurden, waren es in Norwegen 5 und in Dänemark 11. Festzustellen ist 
jedenfalls, dass sich der schwedische Sonderweg wirtschaftlich nicht besonders ausgezahlt hat. Das schwedische Bruttoinlandsprodukt bricht nach Prognosen 2020 ähnlich wie das von Dänemark um fast fünf Prozent ein, während das norwegische BIP laut Prognose um weniger als drei Prozent zurückgeht [9].

Weil die Bundesländer in Deutschland in unterschiedlichem Maße das öffentliche Leben eingeschränkt haben, lassen sich auch hierzulande entsprechende Analysen durchführen. Wiederum kann man das Mobilitätsverhalten der Bevölkerung als $\mathrm{Ma} ß$ der Restriktionen der Entwicklung des Bruttoinlandsprodukts im jeweiligen Bundesland gegenüberstellen. Für das erste Halbjahr 2020 zeigt sich eine negative Korrelation: Wo der Mobilitätsrückgang besonders hoch war, brach das BIP am stärksten ein. Man stellt aber genauso fest: Je höher die Infektionsraten, umso stärker ist der Rückgang des BIP. Es gibt auch noch weitere Korrelationen, sodass die Frage nach den wirtschaftlichen Auswirkungen lokaler Shutdowns aufgrund mehrerer Einflussfaktoren schwer zu beantworten ist.

Das ifo Institut hat in Kooperation mit dem Helmholtz-Zentrum für Infektionsforschung, Braunschweig, in einer Studie untersucht, ob es wirtschaftlich stets günstiger ist, Öffnungsprozesse so zu beschleunigen, dass ein Konflikt zu Gesundheitszielen entsteht [10, 11]. Für die Studie wurden epidemiologische und ökonomische Simulationsmodelle miteinander verknüpft. Das Ergebnis: Eine zu starke Lockerung ohne Kontrolle der Epidemie führt zu mehr COVID-19-Toten, aber auch zu höheren wirtschaftlichen Gesamtkosten als eine moderate, umsichtige Öffnungsstrategie. Selbstverständlich gehen in solche Simulationen bestimmte Annahmen ein, aber das Ergebnis erscheint aufgrund verschiedener Robustheitstest vertrauenswürdig. Daraus lässt sich schließen: Die Annahme, dass grundsätzlich ein Konflikt zwischen Gesundheitszielen und wirtschaftlichen Zielen besteht, ist nach den gegenwärtigen wissenschaftlichen Ergebnissen falsch.

\section{Zusammenfassung}

- Die Coronapandemie stellt die Finanzkrise in den Schatten, voraussichtlich erreicht sie aber nicht die Dimension der Depression in den 1930-er Jahren.

- Die Verbreitung des Virus beeinträchtigt auch ohne staatliche Restriktionen die Wirtschaft. Die Lockdowns verstärken allenfalls kurzfristig den wirtschaftlichen Einbruch.

- Frühzeitige staatliche Restriktionen begrenzen mittelfristigen wirtschaftlichen Schaden.

- Die anrollende zweite Infektionswelle wird die wirtschaftliche Erholung in Deutschland voraussichtlich stoppen.

- Die Stützung der Konsumnachfrage während der Pandemie durch den Staat hilft wenig. Weil die Krise die verschiedenen Sektoren in sehr unterschiedlichem Ausmaß trifft, benötigt die Politik zielgenaue Instrumente. Dazu zählen beispielsweise Überbrückungshilfen oder die Möglichkeit des Verlustrücktrags. Das hieße, dass Unternehmen die jetzigen Pandemie-bedingten Verluste steuerlich mit Gewinnen des vergangenen Jahres verrechnen dürften. 


\section{Literatur}

1. Jordà, Ò., Singh, S.R., Taylor, A.M.: Longer-run economic consequences of pandemics. NBER Working Paper No. 26934. National Bureau of Economic Research (2020)

2. Barro, R.J., Ursúa, J.F., Weng, J.: The coronavirus and the great influenza pandemic: Lessons from the "spanish flu“ for the coronavirus's potential effects on mortality and economic activity. CESifo Working Paper No. 8166 (2020)

3. Wollmershäuser, T.: ifo Konjunkturprognose Herbst 2020: Deutsche Wirtschaft weiter auf Erholungskurs. ifo Schnelld. Digital 1(11) (2020)

4. Sauer, S., Wohlrabe, K.: Konjunkturumfragen im Fokus: Deutsche Wirtschaft in CoronaSchockstarre. ifo Schnelld. 71(4), 44-47 (2020)

5. Statistisches Bundesamt: Außenhandel, Fachserie 7, Reihe 1. August (2020)

6. Wollmershäuser, T., et al.: ifo Konjunkturprognose Winter 2020: Das Coronavirus schlägt zurück - erneuter Shutdown bremst Konjunktur ein zweites Mal aus. ifo Schnelld. 73, Sonderausgabe Dezember 2020, 03-61 (2020)

7. Correia, S., Luck, S., Verner, E.: Pandemics depress the economy, public health interventions do not: Evidence from the 1918 flu. https://doi.org/10.2139/ssrn.3561560

8. Johns-Hopkins-University: Coronavirus resource center. https://coronavirus.jhu.edu/map. html. Zugegriffen: 21. Okt 2020

9. International Monetary Fund (IMF): World economic outlook, October 2020: a long and difficult ascent. Washington D.C. (2020)

10. Dorn, F., Khailaie, S., Stöckli, M., Binder, S., Lange, B., Vanella, P., Wollmershäuser, T., Peichl, A., Fuest, C., Meyer-Hermann, M.: Das gemeinsame Interesse von Gesundheit und Wirtschaft: Eine Szenarienrechnung zur Eindämmung der Corona-Pandemie. ifo Schnelld. Digital 01(06) (2020)

11. Dorn, F., Khailaie, S., Stöckli, M., Binder, S., Lange, B., Lautenbacher, S., Vanella, P., Wollmershäuser, T., Peichl, A., Fuest, C., Meyer-Hermann, M.: The common interests of health protection and the economy: Evidence from scenario calculations of COVID-19 containment policies. https://doi.org/10.1101/2020.08.14.20175224

Open Access Dieses Kapitel wird unter der Creative Commons Namensnennung - Nicht kommerziell - Keine Bearbeitung 4.0 International Lizenz (http://creativecommons.org/ licenses/by-nc-nd/4.0/deed.de) veröffentlicht, welche die nicht-kommerzielle Nutzung, Vervielfältigung, Verbreitung und Wiedergabe in jeglichem Medium und Format erlaubt, sofern Sie den/die ursprünglichen Autor(en) und die Quelle ordnungsgemäß nennen, einen Link zur Creative Commons Lizenz beifügen und angeben, ob Änderungen vorgenommen wurden. Die Lizenz gibt Ihnen nicht das Recht, bearbeitete oder sonst wie umgestaltete Fassungen dieses Werkes zu verbreiten oder öffentlich wiederzugeben.

Die in diesem Kapitel enthaltenen Bilder und sonstiges Drittmaterial unterliegen ebenfalls der genannten Creative Commons Lizenz, sofern sich aus der Abbildungslegende nichts anderes ergibt. Sofern das betreffende Material nicht unter der genannten Creative Commons Lizenz steht und die betreffende Handlung nicht nach gesetzlichen Vorschriften erlaubt ist, ist auch für die oben aufgeführten nicht-kommerziellen Weiterverwendungen des Materials die Einwilligung des jeweiligen Rechteinhabers einzuholen. 